\title{
In situ observation of nanoparticle ordering at the air-water-substrate boundary in colloidal solutions using $x$-ray nanobeams
}

\author{
S. V. Roth, ${ }^{\text {a) }}$ T. Autenrieth, and G. Grübel \\ DESY, Notkestr. 85, D-22607 Hamburg, Germany \\ C. Riekel and M. Burghammer \\ ESRF, 6r Jules Horowitz, F-38043 Grenoble Cedex 9, France \\ R. Hengstler, L. Schulz, and P. Müller-Buschbaum \\ Physik-Department, TU München, LS E13, James-Franck-Str. 1, 85747 Garching, Germany
}

(Received 11 July 2007; accepted 6 August 2007; published online 29 August 2007)

\begin{abstract}
The nanoscale structuring during evaporation of a droplet consisting of an aqueous colloidal solution of $2 \mathrm{~nm}$ gold nanoparticles in water on a silicon substrate is followed in real time. The authors investigated the transfer of lateral order and vertical layering as a function of time at the three-phase contact line air-solution substrate combining a nanometer-sized $\mathrm{x}$-ray beam with a grazing incidence geometry. A pronounced retardation of vertical ordering is observed with respect to lateral ordering. While individual layers are deposited during evaporation of the solvent, the growth parallel to the substrate shows a strongly nondiffusive behavior. (C) 2007 American Institute of Physics. [DOI: 10.1063/1.2776850]
\end{abstract}

Large-scale arrays of ordered nanoparticles are fascinating materials for science and technology, e.g., data storage ${ }^{1}$ or DNA sensoring, ${ }^{2}$ due to their distinct optical properties. ${ }^{2-4}$ To deposit the nanoparticle layer on top of the substrate, several methods are available, e.g., vacuum deposition ${ }^{1,5}$ and solution casting. Solution casting allows nanostructuring of large-area two-dimensional (2D) thin films with specific morphology, offering the possibility to design $2 \mathrm{D}$ or threedimensional photonic crystals. This method is especially important and applicable in the field of colloidal particles, as colloidal particles are often suspended in aqueous solutions.

Nanostructuring, however, is a very complex process involving several mechanisms. ${ }^{6}$ The solvent evaporates and increases the concentration of the colloidal particles. The increased evaporation near the contact line drives a convective flow within the drop that transports material toward the periphery. ${ }^{7}$ Additionally, an increased solute concentration and a decreased temperature near the three-phase contact line (TPCL) may trigger solutal ${ }^{8}$ and thermocapillary ${ }^{9}$ Marangoni flows. Furthermore, the interaction with the substrate ${ }^{10}$ and transversal contact line instabilities ${ }^{11}$ have to be taken into account. Finally, capillary forces come into play as soon as the solution film has a comparable thickness as the colloidal particles' diameter. $^{12}$

Previous studies addressed the ordering of nanoparticles at the liquid-air interface. ${ }^{13}$ However, for technical applications it is of great importance to transfer this order to a solid substrate. ${ }^{14}$ The interaction with the substrate allows for tuning the arrangement of the nanoparticles and thus the layers' optical properties. Hence, it is only natural to investigate in situ the evolution of ordering at the TPCL liquid-air substrate.

Grazing incidence small angle x-ray scattering ${ }^{5,6}$ (GISAXS) has proven to be a well suited technique for realtime studies. Here, the x-ray beam impinges under a small

\footnotetext{
a)Electronic mail: stephan.roth@desy.de; URL: http://www.desy.de/ sroth/ homepage.htm
}

angle $\alpha_{i}<1^{\circ}$ on to the sample surface. ${ }^{6}$ We combined a nanobeam small angle $x$-ray scattering (nano-SAXS) geometry of ID13 /ESRF with a grazing incidence setup, allowing for nanobeam-grazing incidence small angle $\mathrm{x}$-ray scattering (nano-GISAXS) experiments. We used the extremely brilliant $300 \mathrm{~nm}$ size beam (full width at half maximum) produced by Fresnel zone plates at a wavelength of $0.0976 \mathrm{~nm}$. The angle of incidence was $\alpha_{i}=0.543^{\circ}$. The resulting footprint on the sample of $0.3 \times 32 \mu \mathrm{m}^{2}$ reduced the beam size compared to previous microbeam and submicrobeam GISAXS experiments ${ }^{5,6}$ up to a factor of 10 .

The samples consisted of commercial unconjugated colloidal gold solutions of $2 \mathrm{~nm}$ particle size (Kisker Biotechnologie). Gold nanoparticles are inert and provide strong scattering contrast to the solvent (water). A droplet of $25 \mu \mathrm{l}$ volume was deposited on a blank, acid cleaned Si-wafer surface using a high-precision, remote controlled syringe pump. The resulting droplet diameter on the surface was $3 \mathrm{~mm}$. Hence, the surface curvature of the droplet can be neglected and the droplet surface with respect to the beam can be considered as locally flat (see Fig. 1). The beam was positioned at the TPCL. As an example, we focused on a colloidal particle diameter of $2 \mathrm{~nm}$. Figures 1(b)-1(d) shows the different

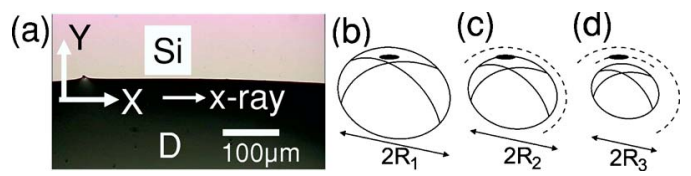

FIG. 1. (Color online) Geometry of the nanobeam-grazing incidence small angle x-ray scattering (nano-GISAXS) experiment: (a) Si denotes the silicon substrate and $D$ the droplet. The beam position and direction $(X)$ is shown as an arrow as well as a scale bar. $X, Y$ denote a coordinate system parallel to the sample surface. Due to an angle of incidence of $\alpha_{i}=0.543^{\circ}$, the beam footprint is $0.3 \times 32 \mu \mathrm{m}^{2}(X \times Y)$. [(b)-(d)] Sketch of the droplet evolution during solvent evaporation. The nanobeam is positioned within $50 \mu \mathrm{m}$ of the initial three-phase contact line (TPCL) after deposition of the droplet. (b) TPCL is far away. (c) TPCL reaches the beam. (d) TPCL has passed the beam. $R_{1}>R_{2}>R_{3}$ during solvent evaporation with $R_{j}(j=1,2,3)$ being the droplet radius. 

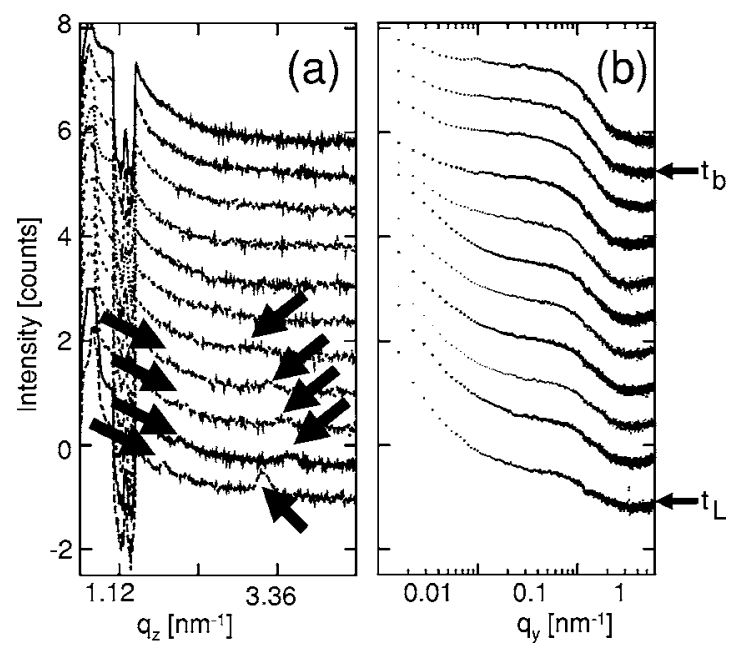

FIG. 2. (a) Detector cut at $q_{y}=0 \mathrm{~nm}^{-1}$. The arrows denote the Bragg peaks due to the thickness of the drying colloidal solution. From top to bottom, the evaporation time increases $\left(t_{b}=3250 \mathrm{~s}\right.$ corresponds to the situation in Fig. 1(c). The time interval between subsequent measurements is $\Delta t=130 \mathrm{~s}$. The curves are shifted for clarity. Bragg peaks become visible for $t=t_{b}+780 \mathrm{~s}$ $=4030 \mathrm{~s}$. (b) Corresponding out-of-plane cut at the critical angle of Si. Clear structural changes occur for $t>t_{b}$ for $q_{y}<0.7 \mathrm{~nm}^{-1}$. For comparison, the lowest curve shows the onset of linear increase time at $t_{L}=t_{b}+2080 \mathrm{~s}$.

stages of solvent evaporation. For the in situ experiment, an acquisition time of $120 \mathrm{~s}$ and a time between frames of $130 \mathrm{~s}$ were chosen. The acquisition of the nano-GISAXS pattern started immediately after casting of the droplet. Figure 2 shows the corresponding results. The 2D nano-GISAXS signal at a sample-to-detector distance $L_{\mathrm{SD}}=0.809 \mathrm{~m}$ is decomposed in two components. The so-called detector $\mathrm{cut}^{5,6}$ shown in Fig. 2(a) probes the intensity distribution as a function of scattering vector component $q_{z}$ (vertical to the sample surface). From bottom to top, the time after deposition increases. In Fig. 2(b), the so-called out-of-plane cut is presented. It shows the intensity distribution $I\left(q_{y}\right)$ of the scattering vector component $q_{y}$ (parallel to the sample surface) and hence probes lateral length scales, e.g., most-prominent radii of clusters.

For times $<t_{b}=3250 \mathrm{~s}$, no change in the nano-GISAXS spectra is observed. At time $t_{b}$, corresponding to Fig. 1(c), the TPCL reaches the beam position and clear changes in the nano-GISAXS pattern occur. From Fig. 2(b), we are able to extract a most-prominent length scale $\xi$ using a Guinier approximation $I\left(q_{y}\right) \propto \exp \left(-\left(q_{y} R_{g}\right)^{2} / 3\right)$ as a function of time within the region $0.037 \mathrm{~nm}^{-1}<q_{y}<0.08 \mathrm{~nm}^{-1}$. Here, $R_{g}$ is the Guinier radius ${ }^{15}$ and $\xi=\sqrt{5 / 3} R_{g}$. The results are shown in Fig. 3(b), with the time $t$ being calculated relative to $t_{b}$, i.e., time after deposition is $t+t_{b}$. At $t=0 \mathrm{~s}$, corresponding to Fig. 1(c), the droplet volume has considerably decreased, and we observe that the lateral growth sets in. $\xi$ increases according to a power law as function of time, $\xi(t) \propto t^{0.32 \pm 0.06}$, indicating that the structure formation is governed not only by diffusive processes, which would lead to $t^{1 / 2}$. Additionally, the detector scan in Fig. 2(a) shows the decreasing solution film thickness, as indicated by the arrows. We analyzed the detector cuts using the distorted wave born approximation which includes refraction and reflection effects. ${ }^{16}$ Following Ref. 17, we estimate the layer thickness by using the formula for the Bragg reflections,

$$
q_{z}=\frac{2 \pi}{\lambda}\left(\sin \left(\alpha_{i}\right)+\sqrt{\sin ^{2}\left(\alpha_{c, \mathrm{Au}}\right)+\left[\mathrm{BL}\left(m, \alpha_{i}, t\right)\right]^{2}}\right),
$$
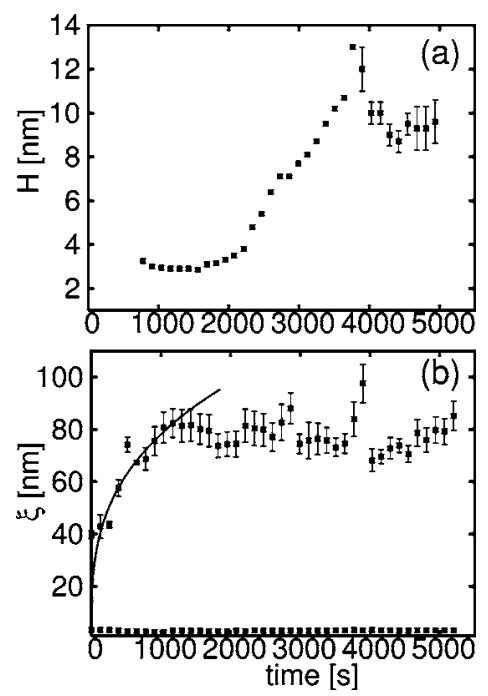

FIG. 3. (a) Quantitative analysis of the length scales in vertical direction as derived from the detector cut Fig. 2(a). The time is calculated relative to $t_{b}$, hence $t=0 \mathrm{~s}$ corresponds to the arrival of the three-phase-contact line at the beam position [see Fig. 1(c)]. For $780 \mathrm{~s}<t<2000 \mathrm{~s}$, the layer thickness, as derived from the Bragg peaks in Fig. 2(a), remains nearly constant. The subsequent increase stems from layer-by-layer deposition (one layer each $340 \mathrm{~s}$ ). After the peak, compaction of the colloidal layer occurs, and the height stays constant. (b) Most-prominent in plane length scale $\xi$. $\xi(t)$ follows a $t^{0.32 \pm 0.06}$ power law (solid line). The lower symbols indicate the radius of the spherical gold nanoparticles. Clearly, the layer-by-layer deposition is retarded with respect to the lateral nanostructuring.

$$
\mathrm{BL}\left(m, \alpha_{i}, t\right)=\frac{m \lambda}{H} \pm \sqrt{\sin ^{2}\left(\alpha_{i}\right)-\sin ^{2}\left(\alpha_{c, \mathrm{Au}}\right)}
$$

where $\alpha_{i}$ denotes the incident angle, $\alpha_{c, A u}$ the critical angle of gold, $H(t)$ the height of the resulting layer as a function of time $t, m$ the order of the Bragg reflection, and $\lambda$ the wavelength. In our case, $\alpha_{i}$ is fixed, and we observe Bragg reflections for different times at different $q_{z}$. As can be seen in Fig. 2(a), higher orders $(m>1)$ can occur. From the fits of the positions of the Bragg peaks in Fig. 2(a) using Eq. (1), we can analyze the height of the resulting nanoparticle layer. The result is shown in Fig. 3(a). For times $t<2000 \mathrm{~s}$, a constant height is found. This seems to correspond to a rapidly deposited, short-range order hexagonal close packing of an $n=2$ layer of colloidal spheres of $2 \mathrm{~nm}$ diameter, yielding a thickness of about $3 \mathrm{~nm}$. For $t>2260 \mathrm{~s}$, which is much larger than the time where lateral growth sets in, a linear growth following $0.0049 \mathrm{~nm} / \mathrm{s} \times t$ in height $H$ of the layers is found. At $t=3770 \mathrm{~s}$ the vertical growth is essentially finished and levels of into its final value $H=9.2 \mathrm{~nm}$ due to compaction. From the linear time dependence of $2080 \mathrm{~s}<t$ $<3770 \mathrm{~s}$, one can estimate the establishment of a full layer with height increase of $2 \mathrm{~nm} \times \sin 60^{\circ}=1.7 \mathrm{~nm}$ every $340 \mathrm{~s}$, assuming close packing of the colloidal particles. This would correspond to a layer-by-layer growth every $340 \mathrm{~s}$ assuming close packing. As one can see the final ordering in this aqueous colloidal solution takes place with time scales on the order of some $10 \mathrm{~s}$. More remarkably, the lateral growth is finished before vertical growth sets in. This time delay between vertical and lateral growth has not been reported yet.

We have for the first time employed nanobeam GISAXS to follow in situ the nanostructuring during solution casting quantitatively. The lateral assembly manifesting itself in the length scale $\xi$ precedes the vertical growth of the nanolayer. 
This structure formation can be interpreted as clustering of nanoparticles and domain formation during evaporation of the solvent. We finally speculate that the nondiffusive character of lateral clustering as indicated by the power-law behavior might be caused by convective flows, reducing the exponent.

This work was financially supported by the DFG Schwerpunktprogramm SPP 1164 "Nano- and Microfluidics" (Mu1487/2).

\footnotetext{
${ }^{1}$ A. Biswas, P. C. Karulkar, H. Eilers, M. Grant Norton, D. Skorski, C. Davitt, H. Greve, U. Schürmann, V. Zaporojtchenko, and F. Faupel, Vac. Technol. Coat. 7, 54 (2006).

${ }^{2}$ G. Bauer, J. Hassmann, H. Walter, J. Haglmüller, C. Mayer, and T. Schalkhammer, Nanotechnology 14, 1289 (2003).

${ }^{3}$ H. Ditlbacher, J. R. Krenn, B. Lamprecht, A. Leitner, and F. R. Aussenegg, Opt. Lett. 25, 563 (2000).

${ }^{4}$ A. Hynninen, J. H. J. Thijssen, E. C. M. Vermolen, M. Dijkstra, and A. V. Blaaderen, Nat. Mater. 6, 202 (2007).
}

${ }^{5}$ S. V. Roth, H. Walter, M. Burghammer, C. Riekel, B. Lengeler, C. Schroer, M. Kuhlmann, T. Walther, A. Sehrbrock, R. Domnick, and P. Müller-Buschbaum, Appl. Phys. Lett. 88, 021910 (2006).

${ }^{6}$ P. Müller-Buschbaum, E. Bauer, S. Pfister, S. V. Roth, M. Burghammer, C. Riekel, C. David, and U. Thiele, Europhys. Lett. 73, 35 (2006).

${ }^{7}$ M. Gonuguntla and A. Sharma, Langmuir 20, 3456 (2004).

${ }^{8}$ V. X. Nguyen and K. J. Stebe, Phys. Rev. Lett. 88, 164501 (2002).

${ }^{9}$ M. D. Haw, M. Gillie, and W. C. K. Poon, Langmuir 18, 1626 (2002); H. Hua and R. G. Larson, ibid. 21, 3963 (2005); 21, 3972 (2005).

${ }^{10}$ M. R. E. Warner, R. V. Craster, and O. K. Matar, J. Colloid Interface Sci. 267, 92 (2003).

${ }^{11}$ R. D. Deegan, Phys. Rev. E 61, 475 (2000).

${ }^{12}$ N. D. Denkov, O. D. Velev, P. A. Kralchevsky, I. B. Ivanov, H. Yoshimura, and K. Nagayama, Nature (London) 361, 26 (1993).

${ }^{13}$ S. Narayanan, Jin Wang, and Xiao-Min Lin, Phys. Rev. Lett. 93, 135503 (2004).

${ }^{14}$ W. Cheng, J. Wang, U. Jonas, G. Fytas, and N. Stefanou, Nat. Mater. 5, 830 (2006)

${ }^{15}$ A. Guinier, Ann. Phys. 12, 161 (1939).

${ }^{16}$ Remi Lazzari, J. Appl. Crystallogr. 35, 406 (2002).

${ }^{17}$ P. Busch, D. Posselt, D.-M. Smilgies, M. Rauscher, and C. M. Papadakis, Macromolecules 40, 1630 (2007). 